\title{
Control of Defects and Spacelike Structures in Delayed Dynamical Systems
}

\author{
S. Boccaletti, ${ }^{1}$ D. Maza, ${ }^{2}$ H. Mancini, ${ }^{2}$ R. Genesio, ${ }^{3}$ and F. T. Arecchi ${ }^{1}$ \\ ${ }^{1}$ Istituto Nazionale di Ottica, I50125 Florence, Italy \\ and Department of Physics, University of Florence, I50125 Florence, Italy \\ ${ }^{2}$ Department of Physics and Applied Mathematics, Universidad de Navarra, Pamplona, Spain \\ ${ }^{3}$ Department of Sistemi e Informatica, University of Florence, 150139 Florence, Italy
}

(Received 30 April 1997; revised manuscript received 18 September 1997)

\begin{abstract}
In many nonequilibrium dynamical situations delays are crucial in inducing chaotic scenarios. In particular, a delayed feedback in an oscillator can break the regular oscillation into trains mutually uncorrelated in phase, whereby the phase jumps are localized as defects in an extended system. We show that an adaptive control procedure is effective in suppressing these defects and stabilizing the regular oscillations. The analysis of the transient times for achieving control demonstrates that stabilization is obtained within an amplitude turbulent regime, analogous to what is present in spatially distributed systems. The control technique is robust against the presence of large amounts of noise. [S0031-9007(97)04933-8]
\end{abstract}

PACS numbers: 05.45. $+\mathrm{b}, 47.52 .+\mathrm{j}, 47.54 .+\mathrm{r}$

Since the original idea of Ott, Grebogi, and Yorke [1], many different theoretical schemes [2] and experimental applications [3] have faced the problem of controlling unstable periodic orbits (UPO's) in chaotic concentrated systems, i.e., in systems modeled by ordinary differential equations.

Some proposals of controlling spatially extended systems, i.e., systems ruled by partial differential equations whose order parameter $y$ is a $m$ dimensional vector $(m \geq$ $1)$ in phase space, with $k$ components $(k \geq 1)$ in real space, have been put forward for the case $k=2$ [4]. However, experimentally implementable tools have not yet been introduced for controlling unstable periodic patterns (UPP) in extended systems.

The essential problems arising in the passage from concentrated to extended systems are already present in delayed dynamical systems, i.e., systems ruled by

$$
\dot{y}=\mathcal{F}\left(y, y_{d}\right) \text {, }
$$

where $y=y(t) \in \mathbb{R}^{m}$, dot denotes temporal derivative, $\mathcal{F}$ is a nonlinear function, and $y_{d} \equiv y(t-T), T$ being a time delay.

Experimental evidence of the analogy between delayed and extended systems was provided for a $\mathrm{CO}_{2}$ laser with delayed feedback [5] and supported by a theoretical model [6]. Most of the statistical indicators for delayed systems, such as the fractal dimensions, are extensive parameters proportional to $T$, which thus plays a role analogous to the size for the extended case [7].

The conversion from the former to the latter case is based on a two variable time representation, defined by

$$
t=\sigma+\theta T,
$$

where $0 \leq \sigma \leq T$ is a continuous spacelike variable and $\theta \in \mathbb{N}$ plays the role of a discrete temporal variable [5]. By such a representation the long range interactions introduced by the delay are reinterpreted as short range interactions along the $\theta$ direction, since now $y_{d} \equiv y(\sigma, \theta-1)$. In this framework, the formation and propagation of space-time structures, as defects and/or spatiotemporal intermittency can be identified $[5,6]$.

When $T$ is larger than the oscillating period of the system, the behavior of a delayed system is analogous to an extended one with $k=1$. In particular, it may display phase defects, i.e., points where the phase suddenly changes its value and the amplitude goes to zero.

In this Letter we introduce a control technique to suppress these defects, stabilizing the oscillations of a delayed system. The control restores regular patterns in two different chaotic regimes, namely, phase turbulence and amplitude turbulence, this last one implying the presence of a large number of defects. The control efficiency persists even in the presence of a large amount of noise.

For the sake of exemplification, we make reference to the following delayed dynamics:

$$
\begin{gathered}
\dot{A}=\varepsilon A+\beta_{1} A^{2}(t-T) A+\beta_{2} A^{4}(t-T) A, \\
\dot{\varepsilon}=\mu\left(S-\frac{\mu_{1}}{\mu} \varepsilon-k A^{2}\right) .
\end{gathered}
$$

Here, all quantities are real. $A$ is an order parameter, $\varepsilon$ is the time-dependent linear gain, $\beta_{1}, \beta_{2}, \mu_{1}, k$ are suitable fixed parameters, $\mu$ is a measure of the ratio between the characteristic time scales for $A$ and $\varepsilon$, and $S$ is a measure of the power provided to the system.

Equations (3) and (4) are rather general. For instance, when $T=0, S<0, \beta_{1}>0, \beta_{2}<0, \mu>0, \mu_{1}>0$, $k>0$ they model an excitable system, producing the so called Leontovitch bifurcation, evidence of which has been shown experimentally on a $\mathrm{CO}_{2}$ laser with intracavity saturable absorber [8]. For $T \neq 0$, they are similar to the models already used to describe self-sustained oscillations of confined jets [9], or memory induced low frequency oscillations in closed convection boxes [10], or even the pulsed dynamics of a fountain [11]. Equations (3) and (4) have been found also to be a good model for the temperature evolution in a well controlled time-dependent convection 
experiment [12]. For convenience we spend a few words to recall the main features of such an experiment, since it provides evidence of the defects we want to control.

A cylindrical layer (diameter $128 \mathrm{~mm}$ ) of silicon oil (depth $15 \mathrm{~mm}$ ) is heated from below by a square heater limited to the central part of the container [side $68 \mathrm{~mm}$, cross section in Fig. 1(a)]. The heater is surrounded by the same insulating material of the vessel. A convective instability driven simultaneously by buoyancy and temperature dependent surface tension ( $80 \%$ and $20 \%$, respectively), called Bénard-Marangoni convection, grows as the heating is increased. A steady state is reached and a stationary pattern composed of four convective cells appears in the hot region. Additional details on this experiment can be found in Ref. [12].

If the heating is further increased, a time-dependent regime arises consisting in spatiotemporal modulations, or thermals, generated at the bottom boundary layer and then dragged by the flow along the cell as can be seen in Fig. 1(a). This configuration provides a natural delayed interaction insofar as it reiterates at each position the local value of the order parameter after a delay $T$, corresponding to the time lag necessary for the trip of the cell. In this situation, an experimental measurement of the temperature at the point $P$ of Fig. 1(a) yields the data of Fig. 1(b). The vertical axis (temperature) is taken as representative of the order parameter $A$. The main feature of this experiment consists of trains of modulated oscillations, interrupted by localized events (phase defects), wherein the phase of the signal changes suddenly and the amplitude decreases to zero.

The relaxation oscillations are represented by the normal form of a Hopf bifurcation [Eq. (3)], in which the saturating terms are delayed to account for the transport of the convective cell. Equation (4) represents the slow evolution $(\mu<1)$ of the control parameter $\varepsilon$, which is enhanced by the external pump $S$ and depressed by the convective motion $\left(-k A^{2}\right)$ which tends to uniformize top and bottom temperatures. Equations (3) and (4) reproduce satisfactorily the experimental signal for rather long delays, and can be considered as an adequate model of the situation we want to control.

The adaptive method we are going to apply is by no means restricted to Eqs. (3) and (4). In fact, it applies successfully to much simpler models as the one in Ref. [6] for a $\mathrm{CO}_{2}$ laser with delayed feedback, which indeed displays phase defects as those reported on Fig. 1.

Let us see how phase defects emerge. We adjust the pump and delay parameters $S$ and $T$ of Eqs. (3) and (4) so that the system enters the chaotic region. This region, in fact, is split into two different regimes. For low $T$ values, chaos is due to a local chaotic evolution of the phase, whereas no appreciable amplitude fluctuations are observed. We call this regime phase turbulence (PT). By increasing $T$, we observe a transition toward amplitude turbulence (AT), wherein the dynamics is dominated by the amplitude fluctuations, and a large number of defects a)

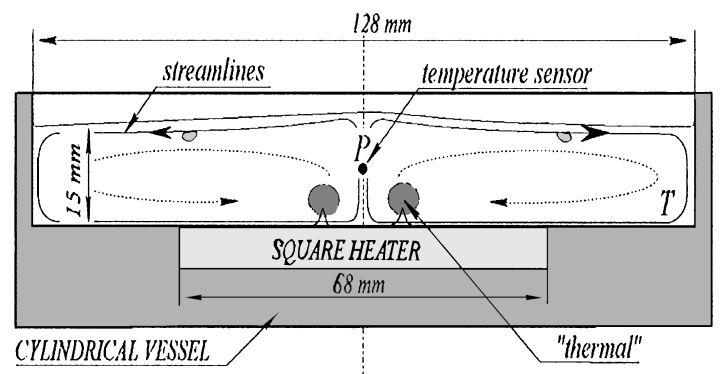

b)

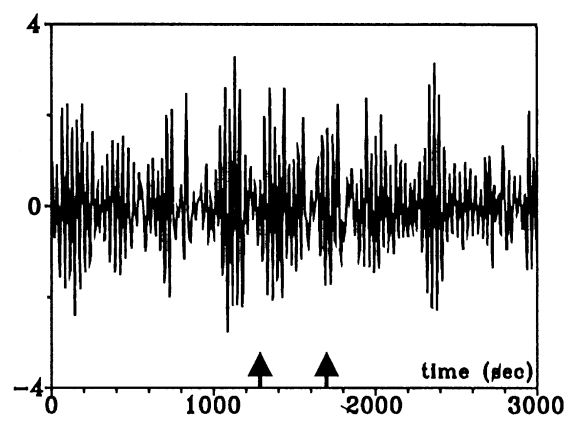

c)

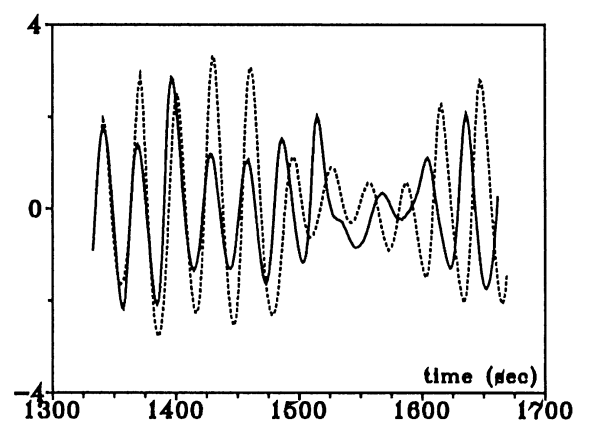

FIG. 1. (a) Cross section of the experimental setup. A hot drop (thermal) is dragged by the flow and then reinjected into the heating region after having completed a round trip of the cell in a mean time $T . P$ indicates the point where temperature is measured. (b) Experimental time behavior of the temperature at the point $P$. Vertical axis reports the temperature in arbitrary units, horizontal axis reports the time in seconds $(T=330 \mathrm{sec})$. (c) Expanded view of the signal within the arrows which exhibits a phase jump (solid line) and reference signal translated by $T$ (dashed line).

is present. Both PT and AT have counterparts in a onedimensional complex Ginzburg-Landau equation. Here the parameter space shows a transition from a regime of stable plane waves toward PT (Benjamin-Fair instability), followed by another transition to AT with evidence of space-time defects [13].

We succeed in controlling both regimes by an adaptive technique recently introduced for chaos recognition [14], and applied to chaos control on concentrated systems [15], chaos synchronization [16], targeting of chaos [17], and filtering of noise from chaotic data sets [18]. This technique adds iteratively a small correction $U(t)$ to Eq. (3), as follows. At time $t_{n+1}=t_{n}+\tau_{n}$ ( $\tau_{n}$ being an adaptive observation time interval to be later specified), the observer defines the variation $A\left(t_{n+1}-T_{H}\right)-A\left(t_{n+1}\right)$ between the actual value of $A$ and the value delayed by the period of the UPO to be controlled ( $T_{H}$ being the Hopf period). The 
corresponding variation rate

$$
\lambda_{n+1}=\frac{1}{\tau_{n}} \log \left|\frac{A\left(t_{n+1}-T_{H}\right)-A\left(t_{n+1}\right)}{A\left(t_{n}-T_{H}\right)-A\left(t_{n}\right)}\right|
$$

allows one to select a new time interval, through the rule

$$
\tau_{n+1}=\tau_{n}\left[1-\tanh \left(g \lambda_{n+1}\right)\right], \quad g>0,
$$

and, consequently, a new observation at the time $t_{n+2}=$ $t_{n+1}+\tau_{n+1}$. The controlling term is given by

$$
U(t)=\frac{1}{\tau_{n+1}}\left[A\left(t-T_{H}\right)-A(t)\right] .
$$

The details of the algorithm have been given in Refs. [14-18]. For practical purposes, the following approximation holds. Let $\langle\tau\rangle$ denote the average of the $\tau_{n}$ set, then Eq. (6) can be written as

$$
\tau_{n+1} \simeq\langle\tau\rangle\left(1-g \lambda_{n+1}\right)
$$

where (i) $\tau_{n}$ has been replaced with its ensemble average, and (ii) the tanh function has been linearized. Point (i) corresponds to fixing once forever a reference time scale for the process under study, while point (ii) corresponds to selecting a conveniently small $g$ to keep $g \lambda_{n+1}$ always within the linear region of the tanh function. In the same way, Eq. (5) can also be linearized as

$$
\lambda(t) \simeq \frac{1}{\langle\tau\rangle} \frac{\dot{A}(t)-\dot{A}\left(t-T_{H}\right)}{A(t)-A\left(t-T_{H}\right)},
$$

where we have further approximated the discretized stroboscopic observations with a continuous inspection. Combining Eqs. (8) and (9) into Eq. (7), this reduces to

$$
\begin{aligned}
U(t)= & K_{1}\left[A\left(t-T_{H}\right)-A(t)\right] \\
& +K_{2}\left[\dot{A}\left(t-T_{H}\right)-\dot{A}(t)\right],
\end{aligned}
$$

with $K_{1}=\frac{1}{\langle\tau\rangle}$ and $K_{2}=\frac{g}{\langle\tau\rangle^{2}}$. The consequences of this approximation are interesting. First of all, for $K_{2}=0$ one recovers the Pyragas control method [19]. However, in our case, $K_{1}$ and $K_{2}$ can be independently selected, and this introduces an extra degree of freedom with respect to Ref. [19]. Now, the control is more active when the error is increasing and vice versa, so reducing oscillations. Indeed, Eq. (10) performs as a proportional derivative controller, the more usual action for stabilizing feedback linear systems, due to its effect which consists of increasing the phase of the compensated system in a suitable frequency band [20].

In Fig. 2 we report the application of our method to Eqs. (3) and (4). The desired oscillation, which in the space-time representation gives rise to a roll set, is controlled in PT [Fig. 2(a)] and in AT [Fig. 2(b)]. Going back to the above discussion, the results show that, while the choice $K_{1}=K_{2}=0.2$ assures the roll stabilization for small perturbations (the $A$ dynamics ranges from 0 to 2 ), fixing $K_{2}=0$ as in the Pyragas' case would have implied prohibitively large $K_{1}$ values for obtaining the same stabilization (in our tests, if $K_{2}=0, K_{1}$ should be 10), resulting in very large perturbations of the system, which eventu-
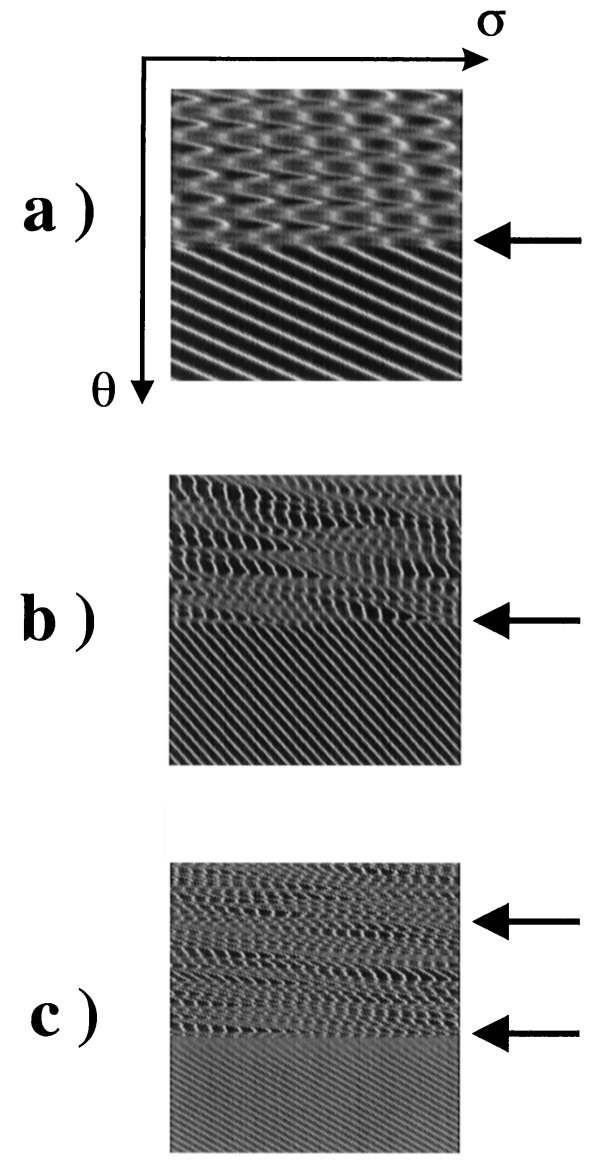

FIG. 2. Space $(\sigma)$-time $(\theta)$ representation of the controlling process for Eqs. (3) and (4). $\beta_{1}=1, \beta_{2}=-1 / 16, \mu=0.8$, $\mu_{1}=0.8, \quad k=11, \quad S=5.5, T_{H}=1.95$. (a) $T=15, \mathrm{PT}$ regime. The chaotic dynamics results in a local turbulent phase of the Hopf oscillation which is corrected by the controlling algorithm. $K_{1}=K_{2}=0.2$. Arrow indicates the instant at which control is switched on. (b) $T=50$, AT regime. The dynamics is dominated by amplitude fluctuations, with the presence of defects. The algorithm $\left(K_{1}=K_{2}=0.2\right)$ suppresses the defects and restores the regular oscillation. Arrow indicates the instant at which control is switched on. (c) Pyragas' method. $T=50$, AT regime. The dynamics is first perturbed with $K_{1}=0.2, K_{2}=0$ (first arrow). To achieve control with $K_{2}=0$ it is necessary to select $K_{1}=10$ (second arrow), which, however, produces a large amplitude distortion (the amplitude of the controlled oscillation is now one half of the amplitude of the Hopf one).

ally give rise to relevant distortions of the roll amplitudes [Fig. 2(c)].

The stabilization consists in suppressing the defects present in the AT regime. Suppose, indeed, that some defects are present at the beginning of the controlling procedure. The spontaneous lifetime $T_{a}$ of a defect can be evaluated in a free running (no control) situation. The scaling behavior of $T_{a}$ as a function of the delay time $T$ depends on the nature of the turbulent process [5]. Namely, in AT, $T_{a}$ scales quadratically with $T$. When a control is applied, we expect it to be effective after a transient time $T_{t}$ of the order of $T_{a}$. Thus a measurement of $T_{t}$ provides an estimate of the lifetime $T_{a}$. 


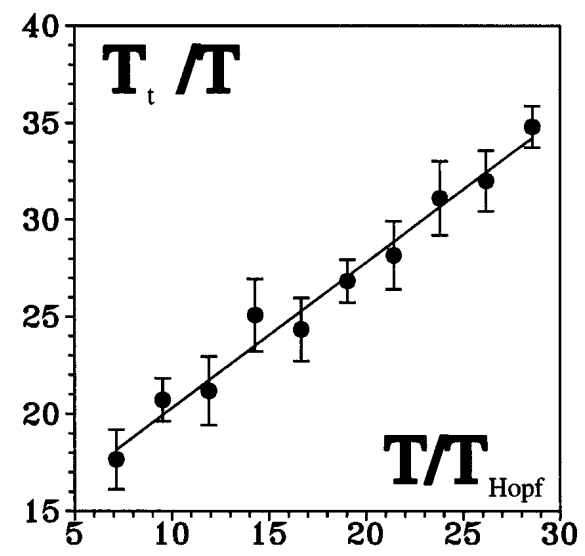

FIG. 3. Plot of the ratio $T_{t} / T$ as a function of $T / T_{H}$ (see text for definitions). The quadratic scaling of $T_{t}(T)$ confirms that control is achieved within AT. Same parameters as in the caption of Fig. 2. For all cases $K_{1}=K_{2}=0.2$.

In Fig. 3 we have reported the scaling behavior of $\frac{T_{t}}{T}$ as a function of $\frac{T}{T_{H}}$. Each point corresponds to a $T_{t}$ value averaged over 20 independent realizations of the control process. The quadratic scaling of $T_{t}(T)$ again confirms that control is achieved within AT.

Finally, let us discuss the robustness of our procedure against external noise. For this purpose, we add white noise to the measured $A$ values before the onset of the adaptive feedback control. Notice that the noise does not act additively, insofar as it is reinjected into the nonlinear
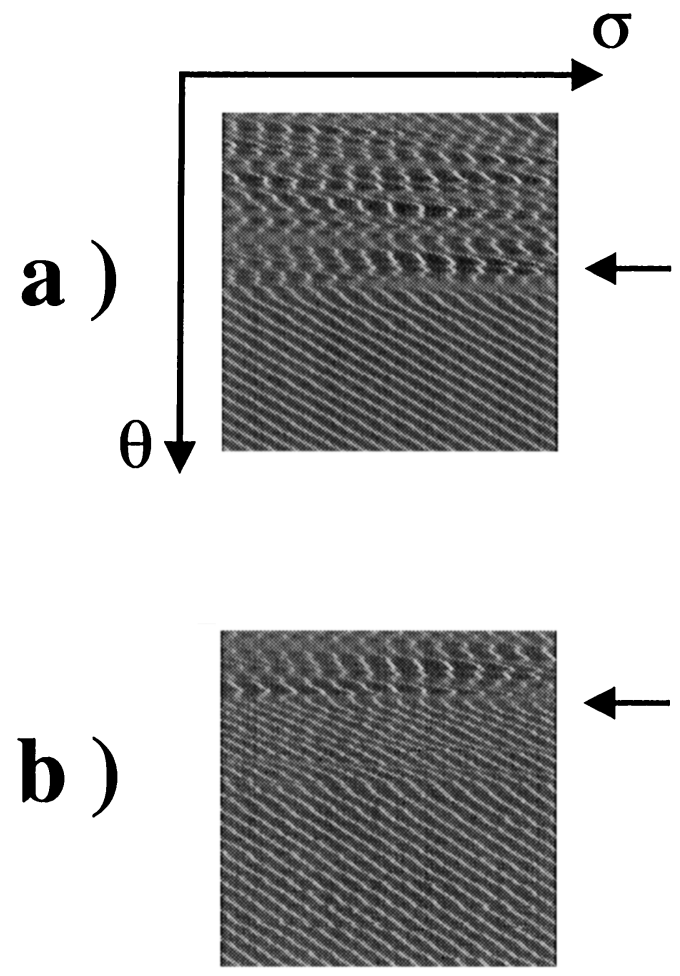

FIG. 4. $T=50$, AT with $10 \%$ noise (a) and $20 \%$ noise (b). Control with $K_{1}=K_{2}=0.2$. Same stipulations and parameters as in the caption of Fig. 2. Arrows indicate the instant at which control is switched on. equations through the control feedback, hence affecting dynamically the evolution of the system. A relevant result is that our method is robust against large amounts of noise. In Fig. 4 the control is achieved within AT for $10 \%$ noise [Fig. 4(a)] and for $20 \%$ noise [Fig. 4(b)]. The controlled UPO is slightly distorted by the action of the noise fed back into the system.

In conclusion, we have introduced a control scheme for stabilizing delayed systems. Its implementation is easy, and experimental application is in progress and it will be reported elsewhere.

Work partly supported by Ministerio de Educacion y Ciencia, Spain (Grant No. PB95-0578), Universidad de Navarra, Spain (PIUNA), European Contract No. ERBFMRX-CT96-0010, and Research Project MURST 40\% "Dynamical Systems and Control."

[1] E. Ott, C. Grebogi, and J. A. Yorke, Phys. Rev. Lett. 64, 1196 (1990).

[2] B. B. Plapp and A. W. Huebler, Phys. Rev. Lett. 65, 2302 (1990); R. Lima and M. Pettini, Phys. Rev. A 41, 726 (1990); A. Azevedo and S. M. Rezende, Phys. Rev. Lett. 66, 1342 (1991); E. R. Hunt, Phys. Rev. Lett. 67, 1953 (1991).

[3] J. Singer, Y.-Z. Wang, and H.H. Bau, Phys. Rev. Lett. 66, 1123 (1991); R. Roy et al., Phys. Rev. Lett. 68, 1259 (1992); V. Petrov et al., Nature (London) 361, 240 (1993); R. Meucci et al., Phys. Rev. E 49, R2528 (1994).

[4] W. Lu, D. Yu, and R. G. Harrison, Phys. Rev. Lett. 76, 3316 (1996); R. Martin et al., Phys. Rev. Lett. 77, 4007 (1996).

[5] F. T. Arecchi et al., Phys. Rev. A 45, R4225 (1992); G. Giacomelli et al., Phys. Rev. Lett. 73, 1099 (1994).

[6] G. Giacomelli and A. Politi, Phys. Rev. Lett. 76, 2686 (1996).

[7] J. D. Farmer, Physica (Amsterdam) 4D, 366 (1982); K. Ikeda and M. Matsumoto, J. Stat. Phys. 44, 955 (1986).

[8] F. Plaza, M. G. Velarde, F. T. Arecchi, S. Boccaletti, M. Ciofini, and R. Meucci, Europhys. Lett. 38, 85 (1997).

[9] E. Villermaux and E. J. Hopfinger, Physica (Amsterdam) 72D, 230 (1994).

[10] E. Villermaux, Phys. Rev. Lett. 75, 4618 (1995).

[11] E. Villermaux, Nature (London) 371, 24 (1994).

[12] H. Mancini and D. Maza, Phys. Rev. E 55, 2757 (1997).

[13] R. Montagne, E. Hernández-Garcia, and M. San Miguel, Phys. Rev. Lett. 77, 267 (1996); A. Torcini, Phys. Rev. Lett. 77, 1047 (1996).

[14] F. T. Arecchi, G. Basti, S. Boccaletti, and A. L. Perrone, Europhys. Lett. 26, 327 (1994).

[15] S. Boccaletti and F. T. Arecchi, Europhys. Lett. 31, 127 (1995); Physica (Amsterdam) 96D, 9 (1996).

[16] S. Boccaletti, A. Farini, and F. T. Arecchi, Phys. Rev. E 55, 4979 (1997).

[17] S. Boccaletti, A. Farini, E. J. Kostelich, and F. T. Arecchi, Phys. Rev. E 55, R4845 (1997).

[18] S. Boccaletti, A. Giaquinta, and F. T. Arecchi, Phys. Rev. E 55, 5393 (1997).

[19] K. Pyragas, Phys. Lett. A 170, 421 (1992).

[20] R.C. Dorf, Modern Control Systems (Addison-Wesley, Reading, MA, 1992), VIth ed. 